\title{
Síndrome do intestino irritável: diagnóstico e tratamento
}

\author{
Irritavel intestinal syndrome: diagnosis and treatment
}

Síndrome del intestino irritable: diagnóstico y tratamiento

Maria Clara Souza Fernandes ${ }^{1 *}$, Mateus de Souza Castro', Yana de Medeiros Souza Lima', Amanda Chagas Barreto ${ }^{1}$, Ângela Milhomem Vasconcelos ${ }^{1}$, Carlos Rafael Alves de Brito ${ }^{1}$, Julia Medeiros Santana ${ }^{1}$, Pâmela Gabrielle Lima Barreiros ${ }^{1}$, Yago Correa Peres ${ }^{1}$, Ana Paula Santos Oliveira Brito'.

\section{RESUMO}

Objetivo: Realizar uma revisão integrativa de artigos publicados em língua inglesa e portuguesa nos últimos sete anos, voltados para as melhores modalidades de diagnóstico e tratamento para abordagem da Síndrome do Intestino Irritável (SII). Métodos: Este estudo constitui-se de uma revisão integrativa, realizada entre janeiro de 2012 e abril 2018, no qual realizou-se uma consulta a livros, periódicos e artigos científicos através de busca no banco de dados do Scientific Electronic Library Online (Scielo) e da Biblioteca Regional de Medicina (Bireme), a partir das fontes Medical Literature Analysis and Retrieval System Online (Medline) e Literatura Latino-americana e do Caribe em Ciências da Saúde (Lilacs). A pesquisa dos artigos foi realizada entre fevereiro e abril de 2018, com o auxílio das palavras-chave Síndrome do Intestino Irritável, Diagnóstico e Tratamento. Resultados: Foram selecionados 26 artigos, realizou-se a análise em busca dos critérios diagnósticos e medidas terapêuticas mais atuais, destes, 10 artigos discorreram sobre as atualizações dos critérios diagnósticos, enquanto 16 artigos analisaram as melhores condutas terapêuticas. Considerações finais: A SII é uma doença heterogênea, a qual deve ser diagnosticada de acordo com os critérios de Roma IV e seus portadores devem receber uma abordagem individualizada, direcionada à melhora na qualidade de vida.

Palavras-chave: Síndrome do intestino irritável, Diagnóstico, Diagnóstico por imagem, Tratamento farmacológico.

\begin{abstract}
Objective: Conduct an integrative review of articles published in English and Portuguese in the last seven years, aimed at the best modalities of diagnosis and treatment for approaching Irritable Bowel Syndrome (IBS). Method: This study consists of an integrative review, carried out between January 2012 and April 2018, in which a consultation of books, journals and scientific articles was carried out by searching the database of the Scientific Electronic Library Online (Scielo) and the Regional Library of Medicine (Bireme), from the sources Medical Literature Analysis and Retrieval System Online (Medline) and Latin American and Caribbean Literature in Health Sciences (Lilacs). The search for the articles was carried out between February and April 2018, with the help of the keywords Irritable Bowel Syndrome, Diagnosis and Treatment. Results: 26 articles were selected, the analysis was carried out in search of the most current diagnostic criteria and therapeutic measures, of these, 10 articles discussed the updates of the diagnostic criteria, while 16 articles analyzed the best therapeutic approaches. Final considerations: IBS is a heterogeneous disease, which must be diagnosed according to the Rome IV criteria and its carriers must receive an individualized approach, aimed at improving quality of life.
\end{abstract}

Keywords: Irritable bowel syndrome, Diagnosis, Diagnostic imaging, Drug therapy.

${ }^{1}$ Centro Universitário Metropolitano da Amazônia (UNIFAMAZ), Belém - PA.

*E-mail: m.claravargens@hotmail.com 


\section{RESUMEN}

Objetivo: Realizar una revisión integradora de los artículos publicados en inglés y portugués en los últimos siete años, con el objetivo de las mejores modalidades de diagnóstico y tratamiento para abordar el Síndrome del Intestino Irritable (SII). Métodos: Este estudio consiste en una revisión integradora, realizada entre enero de 2012 y abril de 2018, en la que se realizó una consulta de libros, revistas y artículos científicos mediante la búsqueda en la base de datos de la Biblioteca Electrónica Científica en línea (Scielo) y el Biblioteca Regional de Medicina (Bireme), de las fuentes Sistema de análisis y recuperación de literatura médica en línea (Medline) y Literatura latinoamericana y caribeña en ciencias de la salud (Lilacs). La búsqueda de los artículos se realizó entre febrero y abril de 2018, con la ayuda de las palabras clave Síndrome de intestino irritable, diagnóstico y tratamiento. Resultados: Se seleccionaron 26 artículos, el análisis se realizó en busca de los criterios diagnósticos y las medidas terapéuticas más actuales, de los cuales 10 artículos discutieron las actualizaciones de los criterios diagnósticos, mientras que 16 artículos analizaron los mejores enfoques terapéuticos. Consideraciones finales: El SII es una enfermedad heterogénea, que debe diagnosticarse según los criterios de Roma IV y sus portadores deben recibir un enfoque individualizado, dirigido a mejorar la calidad de vida.

Palabras clave: Síndrome de intestino irritable, Diagnóstico, Diagnóstico por imagen, Tratamiento farmacológico.

\section{INTRODUÇÃO}

A Síndrome do Intestino Irritável (SII) é uma doença incapacitante, prevalente em toda a população mundial e que causa profundo efeito negativo, na qualidade de vida do indivíduo e nos gastos públicos com saúde. Está descrito que, a nível mundial, entre 10 a $20 \%$ de adultos e adolescentes (1 em cada 5 pessoas) tem sintomas compatíveis com SII. Na América do Sul acomete $21 \%$ da população e no Brasil, aproximadamente, $10 \%$ a $15 \%$ da população, com redução dessa proporção após 60 anos. Ocorre principalmente entre 30 e 50 anos, sendo mais prevalente em mulheres (WGO Global Guidelines, 2015; NADAI R, et al., 2017).

A SII é definida por critérios diagnósticos baseados em sintomas na ausência de causas orgânicas detectáveis, ou seja, entra como diagnóstico diferencial de diversas enfermidades que acometem o sistema gastrointestinal, principalmente de forma crônica. Diante do exposto, é válido ressaltar que a SIl é caracterizada por dor, desconforto abdominal e alterações do hábito intestinal. Além de sensações de desconforto, distensão abdominal e defecação desordenada. (NADAI R, et al., 2017; RODRIGUES GA e CASSIMIRRO RF, 2018).

Destarte, a SII faz parte do grupo dos Distúrbios Funcionais Gastrointestinais (DFGI), o qual é composto por enfermidades crônicas, de etiologia decorrente da combinação de vários fatores e com grande importância para a saúde pública.

A sua fisiopatologia ainda não está totalmente esclarecida, porém, vários mecanismos como a alteração na motilidade gastrointestinal e a hipersensibilidade visceral têm sido frequentemente apresentados para explicá-la. Além disso, as literaturas mais recentes propõem a participação da interação entre sistema imune da mucosa, barreira epitelial e fatores luminais, com alimentos e bactérias intestinais, na fisiopatogenia. (WGO Global Guidelines, 2015).

Novas definições à cerca da desordem foram definidas a partir do Consenso de Roma IV. De acordo com estudo realizado pela New England Jornal of Medicine, tais sinais de alarme seriam a idade acima de 50 anos, mudança recente do hábito intestinal, evidências de sangramento gastrointestinal, dor ao evacuar, perda de peso involuntária e história familiar de câncer colorretal. (RODRIGUES GA e CASSIMIRRO RF, 2018).

Dessa forma, a SII constitui uma enfermidade funcional e, nesse sentido, deve ser diferenciada de outras eventualidades funcionais como a síndrome da dor abdominal funcional, a constipação intestinal funcional e a diarreia funcional crônica, fator crucial na conduta diagnóstica e posterior terapêutica. Perante o exposto, 
pode-se aferir a importância do diagnóstico precoce e correto da enfermidade, para que os pacientes posam ter o devido tratamento buscando o melhor prognóstico possível. (WGO Global Guidelines, 2015; RODRIGUES GA e CASSIMIRRO RF, 2018). À vista disso, este trabalho justifica-se pelo fato de que a SII é uma doença incapacitante, a qual reduz a qualidade de vida e a produtividade no trabalho para o portador, caso não seja corretamente diagnosticada e tratada. Deste modo, este trabalho objetiva realizar uma revisão integrativa de artigos publicados em língua inglesa e portuguesa nos últimos 7 anos, voltados para as melhores modalidades de diagnóstico e tratamento para abordagem da SII.

\section{MÉTODOS}

Foi efetuada uma revisão integrativa da literatura, com intuito de capturar artigos que apresentassem em seus desenhos de estudo correlação com o tema, Síndrome do Intestino Irritável. Foram selecionados estudos publicados de janeiro de 2012 a abril de 2018, em língua inglesa e portuguesa. Os descritores utilizados foram síndrome do intestino irritável, diagnóstico, diagnóstico por imagem e tratamento farmacológico e seus respectivos termos em inglês Irritable Bowel Syndrome, Diagnosis, Diagnostic imaging, Drug therapy.

Essas terminologias estão cadastradas nos Descritores em Ciência da Saúde (DeCS) criados pela Biblioteca Virtual em Saúde. As bases de dados consultadas foram Scientific Electronic Library Online (Scielo), Centro Latino-Americano e do Caribe de Informação em Ciências da Saúde - Biblioteca Regional de Medicina (Bireme), Medical Literature Analysis and Retrieval System Online (Medline), Literatura Latinoamericana e do Caribe em Ciências da Saúde (Lilacs), Pubmed e a biblioteca da Colaboração Cochrane. A pesquisa dos artigos foi realizada entre fevereiro e abril de 2018. A primeira etapa da pesquisa constituiu na busca das bases de dados, por meio do cruzamento dos descritores Síndrome do Intestino Irritável AND Tratamento AND Diagnóstico (Lilacs) e Diagnóstico por imagem AND Tratamento farmacológico AND Irritable Bowel Syndrome (PubMED). Posteriormente foram aplicados os filtros: Anos 2018, 2017, 2016, 2015, 2014, 2013, 2012; Humanos.

Após a leitura de títulos e resumos, adotou-se como os critérios de inclusão para os estudos encontrados foram à abordagem diagnóstica e terapêutica da SII, e estudos comparativos entre estas modalidades. Como critérios de exclusão, adotou-se não analisar artigos com informações incompletas e estudos que relatavam exclusivamente assuntos não relacionados a diagnóstico e tratamento da SII, além de estudos fora do intervalo determinado e sem relevância para a pesquisa. Além desse critério de busca, foram incluídas as Diretrizes mundiais de 2015, 2017 e 2018 relacionada à SII, a Probiótico e Prebiótico e à Dieta e intestino. Ao final, foram lidos os textos na íntegra e, para fins didáticos, o presente trabalho foi dividido nos seguintes temas: Diagnóstico, Tratamento: Terapia não-farmacológica e Terapia farmacológica.

\section{RESULTADOS}

Após aplicados os critérios de elegibilidade, 26 artigos foram identificados no período do estudo, publicados a partir de 2012, sendo 23 destes, publicados a partir de 2015, demonstrando que o tema ainda é atual. Quanto ao local de procedência dos artigos, em sua maioria publicados na América do Norte. Nos 26 artigos, realizou-se a análise de diferentes sistemas em busca dos critérios diagnósticos e medidas terapêuticas mais atuais, destes, 10 artigos (Tabela 1) discorreram sobre as atualizações dos critérios diagnósticos, enquanto que 16 artigos (Tabela 2) analisaram as melhores condutas terapêuticas. (Tabela 1).

Tabela 1 - Artigos selecionados sobre as atualizações dos critérios diagnósticos.

\begin{tabular}{cc}
\hline Abordagens & Quantidade de artigos \\
\hline Comparação entre os critérios de roma & 4 \\
Roma iv & 4 \\
Espectro da escala de bristol & 2 \\
\hline
\end{tabular}

Fonte: Fernandes MCS, et al., 2020. 
Tabela 2 - Artigos selecionados sobre as melhores condutas terapêuticas.

\begin{tabular}{|c|c|}
\hline Terapêutica & $\begin{array}{l}\text { Quantidade de } \\
\text { artigos }\end{array}$ \\
\hline $\begin{array}{l}\text { FODMEPs - fermentáveis, oligossacarídeos, dissacarídeos, monossacarídeos e } \\
\text { polióis }\end{array}$ & 13 \\
\hline Fibras solúveis + Ingesta hídrica & 8 \\
\hline Probióticos & 10 \\
\hline Transplante de microbiota fecal & 1 \\
\hline Abordagem psicológica & 4 \\
\hline Antiespasmódicos & 4 \\
\hline Laxantes & 5 \\
\hline Antidiarreicos & 3 \\
\hline Antidepressivos & 5 \\
\hline Antibiótico & 4 \\
\hline Analgésicos & 1 \\
\hline Antiinflamatório & 1 \\
\hline Opiáceo & 2 \\
\hline
\end{tabular}

Fonte: Fernandes MCS, et al., 2020.

Para avaliação do melhor critério diagnóstico, foram eleitos 4 artigos, os quais possuem como base a comparação entre a série de atualizações dos critérios do Consenso de Roma. Ademais, outros 4 artigos foram selecionados, para o melhor entendimento das novas abordagens, especificamente do Consenso de Roma IV. Por conseguinte, 2 artigos foram selecionados para a compreensão do espectro da escala de Bristol na abordagem do paciente com SII, dispostos no Consenso de Roma IV.

Nos 16 artigos, realizou-se a análise de diferentes modalidades de tratamento, destes 13 artigos falaram a favor da restrição de FODMEPS (fermentáveis, oligossacarídeos, dissacarídeos, monossacarídeos e polióis) da dieta, 8 artigos falaram sobre o aumento do uso de fibras e água, 10 artigos enalteceram o uso de probióticos, 4 artigos discorreram sobre a grande importância do apoio psicológico aos portadores de SII, que vai desde terapia cognitivo comportamental e hipnose até relação medico paciente.

Além disso, agora relacionado à terapêutica farmacológica, 4 artigos mencionaram como primeira linha de tratamento o uso de antiespasmódicos, 5 artigos apoiaram o uso de laxantes e 3 artigos apoiaram os antidiarreicos.

Sobre os antidepressivos, 5 artigos relataram o uso de antidepressivos tricíclicos e de inibidores da receptação de serotonina. Em relação a outras terapias, 4 artigos utilizaram antibióticos, 1 artigo falou sobre o uso de analgésicos, 1 sobre o uso de antiinflamatorios e 2 falaram sobre opiáceos, questionando sua eficácia. E por fim, apenas 1 artigo falou sobre transplante da microbiota fecal (TMF), sugerindo que a restauração da homeostase intestinal através do TMF pode levar a melhora sintomática. 
Figura 1 - Critérios de seleção dos artigos.

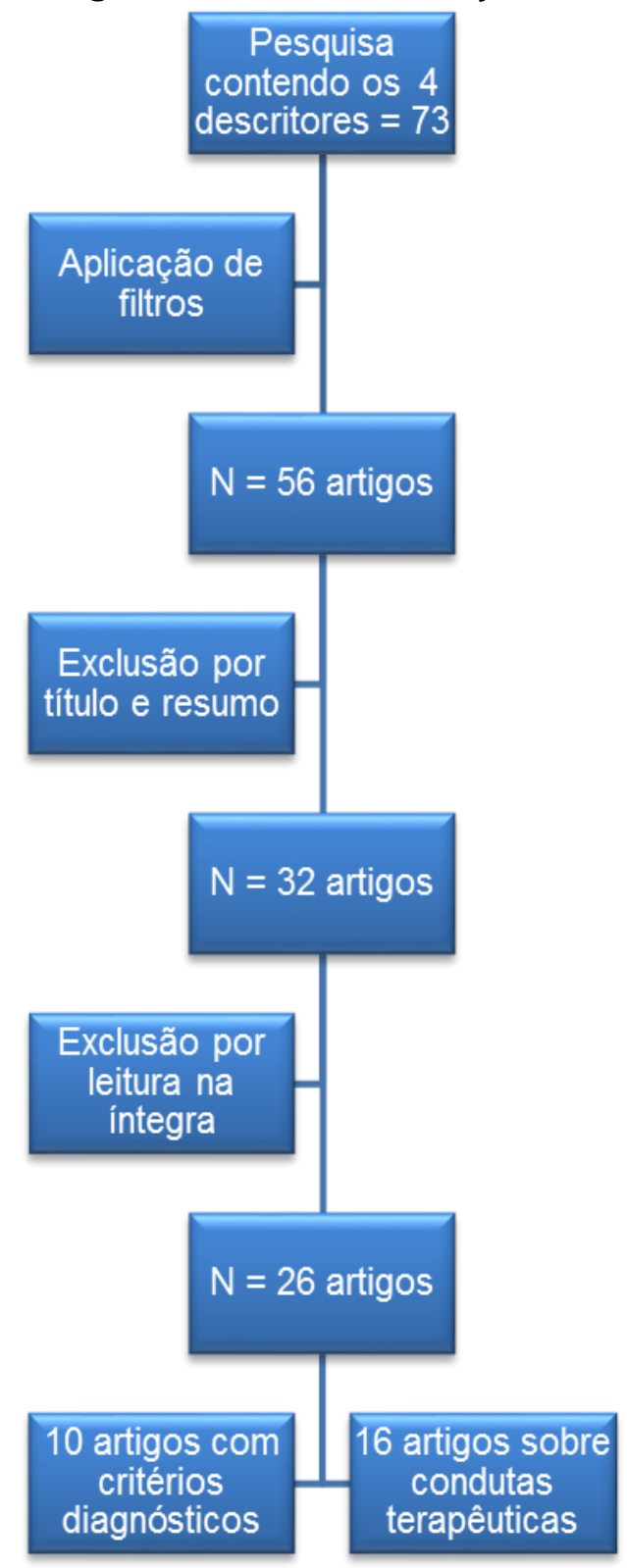

Fonte: Fernandes MCS, et al., 2020.

\section{DISCUSSÃO}

\section{Diagnóstico}

Diante da análise dos estudos dessa revisão, pode-se observar que a síndrome do intestino irritável (SII) é uma condição de alta prevalência, que expressa uma grande dificuldade no diagnóstico, devido possuir um quadro clínico pouco específico, porém com um efeito negativo significativo na qualidade de vida da população, além ser a doença mais representativa do eixo cérebro-intestino. (NADAI R, et al., 2017; JANG SH e KIM YS, 2018).

Estabelecer o diagnóstico da SII pode ser um desafio, já que não há teste confirmatório. Nesse sentido, diagnóstico da SII é alcançado através dos dados clínicos, baseados nos critérios de Roma. Suas versões anteriores consideravam os distúrbios intestinais funcionais, como SII, diarréia funcional, constipação funcional e distensão funcional como entidades separadas (Roma I). Mais tarde, foi reconhecido que esses transtornos poderiam se sobrepor (Roma II e Roma III). (GHOSHAL UC, et al., 2015). 
Dez grandes atualizações foram feitas aos critérios de Roma IV, foram incluídos novos distúrbios por se encaixarem na nova definição de DFGI, são estes, hiperalgesia gastrintestinal induzida por opióides, constipação induzida por opióides e hiperemese canabinóide.

Ademais, os subtipos de distúrbios intestinais (isto é, constipação, diarreia, mistos, não classificados) são vistos em um espectro e não como distúrbios separados, de modo a explicar as diferenças na intensidade, quantidade e gravidade dos sintomas descritas pelos pacientes. (JANG SH e KIM YS, 2018; CHANG L, et al., 2017).

Nesse sentido, o Roma IV introduziu mudanças nos critérios diagnósticos com base em evidências mais recentes. Em primeiro lugar, o termo desconforto presente nos critérios de Roma III é eliminado do critério de Roma IV, agora, dor é usado para estabelecer diagnóstico. Além disso, o limiar para a dor aumentou de 3 dias por mês, para 1 dia por semana, mantida nos últimos 3 meses e recorrente há mais de 6 meses, associado a dois ou mais dos seguintes: piora ou melhora da dor durante a evacuação; mudança na frequência das evacuações; e alteração na Escala de Bristol (EB). (GHOSHAL UC, et al., 2015; CHANG L, et al., 2017; SCHMULSON MJ e DROSSMAN DA, 2017).

Escala de Bristol (EB) é uma classificação formada por sete imagens com características descritivas das fezes, de acordo com a forma e consistência. De acordo com esta escala é correlacionado o tempo de transito intestinal total, medido por cintilografia ou com marcadores radiopacos, com a consistência das fezes, tanto em pacientes com síndrome do intestino irritável como em sujeitos sãos. Dessa maneira, foi demonstrado que os tipos 1, 2 e 3 se correlacionam com tempos de transito lento e o tipo 6 e 7 com tempo de transito rápido. (DALL'AGNOL T e ARAÚJO MP, et al., 2016).

Segundo os Critérios de Roma IV, a SIl pode ser dividida de acordo com as características das fezes, devendo ser usado para descrever objetivamente os hábitos intestinais e classificar os pacientes no subtipo correto, a fim de direcionar o tratamento de acordo com o sintoma predominante, de acordo como é definido pela Escala de Bristol: SII com diarreia (SII-D): Fezes amolecidas > 25\% das vezes e fezes endurecidas < $25 \%$ das vezes, sendo mais frequente entre homens; SII com constipação (SII-C): Fezes endurecidas > $25 \%$ das vezes e fezes amolecidas $<25 \%$ das vezes, sendo mais frequente entre mulheres; SIl com hábitos intestinais mistos ou padrões cíclicos (SII-M): Fezes amolecidas e endurecidas $>25 \%$ das vezes; Nenhum subtipo da SII: Anomalia insuficiente na consistência das fezes para os critérios de SII-C ou M. (MEARIN F, et al., 2016; SPERBER AD, et al., 2016; VORK L, et al., 2018; LACY BE e PATEL NK, 2017).

Em relação as manifestações clínicas dos pacientes com a SII, essa enfermidade pode ser classificada de acordo com os sintomas, em SII com predomínio da disfunção intestinal, SII com dor predominante e SII com distensão abdominal predominante. Além disso, o distúrbio pode ser classificado também de acordo com os fatores precipitantes, como pós-infecciosa (SII-PI), induzida por alimentos (induzida pelas refeições) e relacionada ao estresse. (SPERBER AD, et al., 2016; VORK L, et al., 2018; LACY BE e PATEL NK, 2017).

Nesse sentido, é válido ressaltar que a SIl entra como diagnóstico de exclusão entre outras comorbidades funcionais, e para sua confirmação, deve-se realizar os exames orientados pela anamnese. Caso haja presença de sinais de alarme, se faz necessário a colonoscopia a fim de excluir causas orgânicas (LACY BE, et al., 2016; LACY BE e PATEL NK, 2017).

A colonoscopia permite a visualização direta do cólon bem como a realização de biópsia e atualmente destaca-se como um dos métodos mais completos de investigação das doenças colorretais, incluindo as doenças inflamatórias intestinais. Em alguns pacientes com diarreia crônica, a colonoscopia pode evidenciar mucosa normal, nesses casos a execução de biópsias seriadas pode trazer informações importantes para 0 diagnóstico e tratamento dos pacientes. (OLIVEIRA AR, et al., 2019).

\section{Tratamento}

O tratamento da SIl depende da apresentação clínica, porém, em razão dos diferentes mecanismos fisiopatológicos abordados anteriormente e da heterogeneidade sintomatológica, as medidas devem ser individualizadas. (WGO Global Guidelines, 2015; BASTOS TFS, 2016; MAQUES AM, 2012). 
A abordagem terapêutica baseia-se, sobretudo, na sintomatologia predominante, no grau de comprometimento funcional e nos fatores psicossociais envolvidos. Sendo assim, o tratamento tem como base o alívio sintomático e não a alteração do mecanismo fisiopatológico subjacente. (WGO Global Guidelines, 2015; NADAI R, et al., 2017; RODRIGUES GA e CASSIMIRRO RF, 2018).

O manejo terapêutico abrange tanto categorias farmacológicas, como não farmacológicas. A terapia farmacológica é bastante diversificada, mas a primeira opção são as medidas não farmacológicas. (WGO Global Guidelines, 2015; NADAI R, et al., 2017; SCHMULSON MJ e DROSSMAN DA, 2017).

\section{Terapia não-farmacológica}

Uma modificação dietética que está se mostrando eficaz na terapêutica da SII é a redução da ingesta de grupos alimentares de alta fermentação e efeitos osmóticos, também chamados de FODMEPs, que por serem pequenas moléculas osmoticamente ativas, apresentam má absorção intestinal e rápida fermentação pela microbiota, provocando distensão luminal, em virtude da maior produção de gases hidrogênio e metano, além de maior fornecimento de água para o cólon; desencadeando sintomas gastrointestinais.

Os estudos mostram que, após 24 a 48 horas do início da dieta, tanto portadores da SIl como pessoas saudáveis experimentam diminuição na produção de gases colônicos. (WGO Global Guidelines, 2018; BASTOS TFS, 2016; EL-SALHY M e GUNDERSEN D, 2015; GIBSON PR, et al., 2015; WHIGHAM L, et al., 2015; LACY BE, 2015).

Outra alteração na dieta é que ela seja rica em fibras ou formadora de volume (p. ex., psyllium) combinado com ingestão suficiente de líquidos. Porém, as fibras insolúveis podem exacerbar os sintomas e aliviar pouco e por outro lado, as fibras solúveis como o psyllium (ispagula), aliviam a SII. (WGO Global Guidelines, 2015; HALMOS EP, et al., 2014; AHMAD OF e AKBAR A, 2015; EL-SALHY M e GUNDERSEN D, 2015; EL-SALHY M, 2015; LACY BE, 2015).

Metanálises recentes confirmam o papel dos probióticos, porém expressam que a eficácia da terapêutica dependem altamente da cepa. A cepa probiótica Bifidobacterium infantis 35624 (uma cápsula por dia) tem demonstrado reduzir dor, inchaço e dificuldade na defecação, além de normalizar o trânsito intestinal nos pacientes com SII, independentemente do hábito predominante; mas atualmente está disponível somente nos Estados Unidos, Reino Unido e Irlanda.

Já a cepa probiótica Bifidobacterium lactis DN-173010 tem demonstrado acelerar o trânsito gastrointestinal e aumentar a frequência das evacuações nos pacientes com SIl com constipação. Entretanto, por conta da variabilidade e formulação das cepas não existe, no momento, suficiente evidência para uma recomendação geral dos probióticos em pacientes com SII. (WGO Global Guidelines, 2017; BASTOS TFS, 2016; AHMAD OF e AKBAR A, 2015; EL-SALHY M, 2015; LACY BE, 2015).

Nos pacientes com a síndrome, ocorre diminuição de Lactobacillus e Bifidobacterium e um acréscimo de Enterobacteraceae spp quando confrontado com os controles. Além disso, já foi identificado que existe divergência na constituição da microbiota até mesmo dentre os subtipos de SII. Isso insinua que a reconstituição da homeostase intestinal através do transplante de microbiota fecal (TMF) pode levar a melhoria sintomática. (NADAI R, et al., 2017; WGO Global Guidelines, 2017).

É importante salientar que além das terapêuticas descritas acima, é necessário que intervenções psicológicas possam ser consideradas. Essas abordagens podem incluir: Terapia cognitiva comportamental (TCC), técnicas comportamentais que procuram modificar as condutas disfuncionais (através de técnicas de relaxamento, manejo das contingências e treinamento assertivo).

Também pode ser incluída a hipnose (dirigida ao trato digestivo, recomendada para pacientes com SII refratário ao tratamento medicamentoso convencional), que possui níveis de segurança e tolerabilidade altos, com evidências de eficácia sustentada. Porém, o uso rotineiro é limitado pela dificuldade de conseguir profissionais com tal capacidade e pelo trabalho intensivo requerido. (WGO Global Guidelines, 2015; MAQUES AM, 2012). 


\section{Terapia farmacológica}

Diversos fármacos são usados mundialmente para tratamento das manifestações clínicas individuais da SII. Os principais medicamentos de primeira linha são antiespasmódicos (otilônio, hioscina, cimetrópio, pinavério, diciclomina, mebeverina e o óleo de hortelã) os quais oferecem alívio da dor abdominal. (BASTOS TFS, 2016; MAQUES AM, 2012). Como terapia de segunda linha são usados laxantes como, por exemplo, Lubiprostone ( $8 \mu \mathrm{g} / 2 \mathrm{x}$ ao dia, um agonista dos canais de cloro) ou Linaclotide $(290 \mu \mathrm{g} / 1 \mathrm{x}$ ao dia, 30 minutos antes dos alimentos, um agonista da guanilato ciclase) e antidiarreicos como, por exemplo, Loperamida (2 mg todas as manhãs ou duas vezes ao dia), todos usados para constipação. (BASTOS TFS, 2016; MAQUES AM, 2012; VANUYTSEL T, et al., 2014; BORTOLI N, et al., 2015).

Para o tratamento da dor nesses pacientes, são utilizados antidepressivos tricíclicos (ADT), por exemplo: Amitriptilina (dose inicial $10 \mathrm{mg} / \mathrm{dia}$; dose alvo 25-50 mg/dia, ao deitar) ou Desipramina (dose inicial 50 $\mathrm{mg} / \mathrm{dia}$; dose alvo 100-150 mg/dia, ao deitar) e serotoninérgicos, por exemplo: Paroxetina (10-60 mg/dia) ou Citalopram (5-20 mg/dia). Os receptores da 5-hidroxitriptamina (5-HT), ou receptores da serotonina, estão envolvidos na modulação de múltiplas funções motoras e sensoriais do trato intestinal. Os antagonistas dos receptores 5-HT3 retardam o trânsito intestinal, reduzem a secreção e aumentam a complacência cólica em resposta à distensão. Já os agonistas dos receptores da $5-\mathrm{HT} 4$, diminuem o tempo de trânsito intestinal por apresentarem uma atividade pró cinética com estimulação do peristaltismo. (BASTOS TFS, 2016; MAQUES AM, 2012; VANUYTSEL T, et al., 2014; BORTOLI N, et al., 2015).

A vigência de algumas opções de terapia com Rifaximina (antibiótico de má-absorção com posologia de $550 \mathrm{mg} / 3 \mathrm{x}$ ao dia, durante 14 dias), eficaz para a melhora geral dos sintomas na SII-D. É principalmente bem tolerada em pacientes idosos e mulheres, pois tiveram as taxas mais altas de resposta, mas sua eficácia e segurança não foram estabelecidas além das 16 semanas. Entretanto, recentemente foi comunicada a eficácia e segurança da repetição do tratamento. (BASTOS TFS, 2016; MAQUES AM, 2012).

Outro fármaco que é útil como terapia de segunda linha para SII-D é o Alosetron. Porém, existe associação do medicamento com aumento do risco de colite isquêmica e pode provocar constipação severa. Já na terapia com o Ondansetron foi identificado uma melhora na sensação de urgência, diarreia e inchaço na SII-D, porém não houve melhora no aliviou a dor. O Ramosetron também deveria ser considerado como terapia de segunda linha na SII-D e parece estar desprovido de efeitos adversos graves, como constipação severa e colite isquêmica. (WGO Global Guidelines, 2015; BASTOS TFS, 2016). Para dor também podem ser usados, se for necessário, um analgésico preferivelmente antes dos antiinflamatórios não esteroides (AINEs). Opiáceos devem ser evitados a todo custo, porque a dependência e adição constituem um alto risco nessa afecção crônica. (WGO Global Guidelines, 2015; BASTOS TFS, 2016).

\section{CONSIDERAÇÕES FINAIS}

Diante do exposto, conclui-se que a síndrome do intestino irritável é uma doença heterogênea, a qual deve ser diagnosticada de acordo com os critérios de Roma IV e seus portadores devem receber uma abordagem individualizada, direcionada à melhora na qualidade de vida. Uma abordagem inicial não medicamentosa, com mudança na alimentação, prática de atividades físicas e prática de terapias psicológicas é preconizada. No cenário brasileiro, a abordagem terapêutica para a SII ainda não foi muito explorada, o que é notabilizado pela escassez de estudos acerca do tema. À medida que novas evidências se tornam disponíveis, futuras atualizações são esperadas.

\section{REFERÊNCIAS}

1. AHMAD OF, AKBAR A. (2015). Dietary treatment of irritable bowel syndrome. British Medical Bulletin, 113(1), 8390

2. BASTOS TFS. Síndrome do Intestino Irritável e Dieta com restrição de FODMAPs. Faculdade de Medicina da Universidade de Lisboa. Trabalho Final de Mestrado Integrado em Medicina. 2016.

3. BORTOLI N, et al (2015). Optimal management of constipation associated with irritable bowel syndrome. Ther Clin Risk Manag, 11, 691-703 
4. CHANG L, et al. Updates to the Rome Criteria for Irritable Bowel Syndrome. Gastroenterol Hepatol (NY). $2017 ; 13$ (5): 304-306.

5. DE OLIVEIRA AR, et al. A importância da colonoscopia nas doenças inflamatórias intestinais. Revista de Saúde. 2019 Jan./Jun.;10 (1) Suplementos: 08-12.

6. EL-SALHY M. (2015). Recent developments in the pathophysiology of irritable bowel syndrome. World Journal of Gastroenterology: WJG, 21(25)

7. EL-SALHY M, GUNDERSEN D. (2015). Diet in irritable bowel syndrome. Nutrition Journal 36- 45

8. GHOSHAL UC, et al. Development, Translation and Validation of Enhanced Asian Rome III Questionnaires for Diagnosis of Functional Bowel Diseases in Major Asian Languages. J Neurogastroenterol Motil 2015; 21(1): 83-92

9. GIBSON PR, et al (2015). Food Components and Irritable Bowel Syndrome. Gastroenterology, 148(6), 1158-1174

10. HALMOS EP, et al. (2014) A diet low in FODMAPs reduces symptoms of irritable bowel syndrome. Gastroenterology 146, 67-75

11. DALL'AGNOL T, ARAÚJO MP, LAINO F, PARMIGIANO TR, GIRÃO MJBC, SARTORI MGF. Avaliação do habito intestinal em mulheres atletas e sua relação com nível de hidratação e uso de suplemento. Revista Brasileira de Nutrição Esportiva, São Paulo. v. 10. n. 58. p.458-466. Jul./Ago. 2016. ISSN 1981-9927.

12. JANG SH, KIM YS. Could Improvement in Subjective Well-being Relieve Gastrointestinal Symptoms in Patients With Irritable Bowel Syndrome? Journal of Neurogastroenterology and Motility 2018; 24(2): 164-165

13. LACY BE. (2015). The Science, Evidence, and Practice of Dietary Interventions in Irritable Bowel Syndrome. Clinical Gastroenterology and Hepatology, (April), 1-8 .

14. LACY BE, et al. Bowel Disorders. Gastroenterology. 2016;150:1393-1407

15. LACY BE, PATEL NK. Rome Criteria and a Diagnostic Approach to Irritable Bowel Syndrome. Weber HC, ed. Journal of Clinical Medicine. 2017;6(11):99.

16. MAQUES AM. Síndrome do Intestino Irritável Fisiopatologia e Abordagem Terapêutica. Artigo de Revisão Bibliográfica. Mestrado Integrado em Medicina. 2012.

17. MEARIN F, et al. Bowel Disorders. Gastroenterology. 2016;150:1393-1407. e5.

18. MURRAY K, et al (2014). Differential Effects of FODMAPson Small and Large Intestinal Contents in Healthy Subjects Shown by MRI. The American Journal of Gastroenterology, 109(1), 110-11

19. NADAI R, et al. Transplante de microbiota fecal no tratamento da síndrome do intestino irritável: uma revisão sistemática. Arq Med Hosp Fac Cienc Med Santa Casa São Paulo 2017;62(3):156-9

20. PALSSON OS, et al. Development and validation of the Rome IV diagnostic questionnaire for adults. Gastroenterology, 2016; 150 (6) , pp. 1481-1491.

21. RODRIGUES GA, CASSIMIRRO RF. O uso de probióticos no alívio dos sintomas dos portadores da síndrome do intestino irritável. Revista Brasileira de Ciências da Vida, [S.I.], v. 6, n. 3, abr. 2018.

22. SCHMULSON MJ, DROSSMAN DA. What Is New in Rome IV. Journal of Neurogastroenterology and Motility. 2017;23(2):151-163.

23. SPERBER AD, et al. The global prevalence of IBS in adults remains elusive due to the heterogeneity of studies: a Rome Foundation working team literature review. Jun;66(6):1075-1082. Epub 2016

24. VANUYTSEL T, et al (2014). Treatment of abdominal pain in irritable bowel syndrome. Journal of Gastroenterology, 49, 1193-1205

25. VORK L, et al. III vs Rome IV criteria for irritable bowel syndrome: A comparison of clinical characteristics in a large cohort study. Neurogastroenterol Motil. 2018 Feb;30(2). PMID: 28804974.

26. WHIGHAM L, et al (2015). Clinical effectiveness and economic costs of group versus one-to-one education for short-chain fermentable carbohydrate restriction (low FODMAP diet) in the management of irritable bowel syndrome. J Hum Nutr Diet, 10-20

27. World Gastroenterology Organisation Practice Guidelines. Dieta e Intestino, 2018. WGO Global Guidelines, 2018

28. World Gastroenterology Organisation Practice Guidelines. Probióticos e Prebióticos, 2017. WGO Global Guidelines, 2017

29. World Gastroenterology Organisation Practice Guidelines. Síndrome do intestino irritável: uma Perspectiva Mundial, 2015. WGO Global Guidelines, 2015. 